\title{
Fostering Clinical Judgment Abilities during Clinical Remediation: A Multi-Modal Approach
}

\author{
Cynthia Fenske \\ Campus Dean and Associate Professor \\ Concordia University Ann Arbor \\ cindy.fenske@cuaa.edu \\ Deborah Price \\ Clinical Assistant Professor \\ University of Michigan School of Nursing, Ann Arbor Michigan \\ debprice@umich.edu
}

\begin{abstract}
Remediation has been used for students at risk for clinical failure, with the belief that additional support will enhance the potential for students to be successful their clinical practicums. A multi-modal remediation program was developed which utilized evidence-based approaches to address clinical judgment abilities for students unable to meet the clinical performance objectives. This program incorporated various facets shown to have a positive effect on the development of clinical judgment, including: 1) an individualized remediation plan; 2) low and high fidelity simulation; 3) student self-assessment of their performance compared to actual performance; 4) the Lasater Clinical Judgment Rubric (LCJR) and; 5) a virtual community (VC). This multi-modal approach to remediation appeared to successfully meet the student's learning needs while providing a clearly defined process that used a pedagogically sound framework. The individualized learning plan provided the student with a clear action plan. Simulation provided experiences in a safe environment. The LCJR worked well as a tool for student self-assessment and faculty feedback. The VC aided the students in the 'knowing' of the character as a person and their unique patterns related to their illnesses. This multi-modal remediation program allowed students a chance for success in meeting clinical performance objectives.
\end{abstract}

Keywords: Remediation, Clinical judgment, Nursing students, Self-assessment, Virtual community, Lasater Clinical Judgment Rubric

\section{INTRODUCTION}

Clinical education is a highly complex facet of nursing education in which faculty must facilitate student learning while also maintaining high standards of nursing practice [1]. In order to assure high standards of care, it is imperative that nursing students develop sound clinical judgment abilities, as well as an awareness of the deficits in their clinical practice. Faculty are challenged to develop new learning experiences that will foster the development of clinical judgment abilities by moving students' thinking along the developmental continuum [2]. This becomes even more important when students experience difficulties in achieving the clinical learning objectives, have rudimentary clinical judgment abilities and are at a risk of failing. We sought to develop a multi-modal remediation program which utilized evidence based approaches to address clinical judgment abilities for students unable to meet the clinical performance objectives at any point during their baccalaureate nursing clinical practicums. Remediation has been used for students at risk for clinical failure, with the belief that additional support and resources will enhance the potential for students to be successful in meeting clinical objectives [1]. Remediation for this program was defined as activities intended to meet students' needs when they did not possess the clinical judgment abilities necessary to perform at the required level.

Elements that have been found to contribute to poor student clinical performance include under developed clinical judgment abilities, a lack of hands on clinical experience, a one size fits all clinical instruction, and an unrealistic view of their own abilities $[3 ; 4 ; 5]$. In order to address the elements leading to poor performance, a multi-modal remediation program was developed that incorporated into it the various facets that have been shown to have a positive effect on the development of clinical 
judgment. The program included the following: 1) an individualized remediation plan; 2) low and high fidelity simulation; 3) student self-assessment of their performance compared to their actual performance; 4) the Lasater Clinical Judgment Rubric (LCJR) and; 5) a virtual community (VC). Identifying these five key components was critical to ensure that the program encompassed multiple measures that supported the development of clinical judgment abilities and would ultimately aid the student in meeting their clinical learning objectives.

The Clinical Judgment Model developed by Tanner [6] served as the theoretical foundation of the program. Tanner [6] defines clinical judgment as encompassing the noticing of a problem, an interpretation of the situation based on salient information, the response or action taken within the situation, and the reflection or deconstruction of the judgment used in the situation [6;7]. Research on this topic indicates that clinical judgment develops over time with increased clinical knowledge and experience, and with reflection on one's own clinical judgment in various situations [6;8]. Additionally, experience and expertise in clinical performance is noted to increase clinical judgment abilities in the areas of recognizing patterns or the context of patient responses, developing a sense of salience, using intuition to make decisions and responding rapidly to changes in the patient's condition [6; 8]. Knowing the patient also emerged as a factor in clinical judgment/decision making. In this case knowing is an understanding of the patient as a person and of the patient's patterns of responses. When the nurse is familiar with a patient's usual patterns of responses, salient issues become more apparent as the current situation is compared to this patient's typical presentation [9]. The multi-modal remediation program sought to incorporate these factors throughout its various parts.

The majority of articles about remediation present a single aspect of the teaching plan, including simulation, learning contracts, case-based learning and individualization to facilitate student success in meeting clinical learning outcomes. $[1 ; 10 ; 11 ; 12]$. Each of these programs demonstrated various indictors of success, either with student completion of the remediation program, or with meeting clinical objectives. No studies were found that encompassed a multi-modal approach or included selfassessment, the use of the LCJR, or a virtual community in their program.

\subsection{Individualized learning plan}

An individualized learning plan has been shown to be a strength in the remediation process and allows remediation to leverage the students' motivation for success [13]. Learning methods can be scaffolded to address individualized learning objectives with individualized scenarios which address a specific skills set and learning needs [14]. In this way remediation strategies become more effective when viewed as opportunities that can successfully bridge the student's deficiencies in practice [15]. Clearly identifying and documenting the areas needing remediation has been found to be beneficial for both students and clinical faculty alike as it enables the student to receive a faculty developed action plan identifying areas of their clinical judgment that require remediation $[1 ; 13]$. Gallant et al. [1] found that students are able to use the plan to clearly see, review, and respond to the evidence while decreasing perceived evaluator bias. Faculty supported the individualized approach as it clarified performance expectations and consequences for the student which is helpful in fostering student success $([1 ; 13 ; 15]$.

\subsection{Simulation}

Simulation, both low and high fidelity, is a tool that provides a safe, non-threatening environment for students to practice and test themselves in abilities, while increasing confidence, competence, decision making ability, and clinical judgment abilities $[10 ; 11 ; 16 ; 17 ; 18 ; 19]$. Benner [8] describes simulation as one method that can allow the participant to practice the clinical judgment abilities that fall under effective noticing, interpreting, and responding. Debriefing at the end of the simulation allows for reflecting on performance which is a key factor in clinical judgment development. This gives the participant the opportunity to deconstruct their performance and discuss the strengths of their performance along with the identification of any missed cues, inaccurate priority setting, and inappropriate actions $[7 ; 8 ; 20 ; 21]$.

\subsection{Self-assessment}

Self-awareness of the level of one's own clinical judgment abilities is rarely discussed, but it is important for students to accurately know the level of their abilities. An essential part of developing clinical judgment is being able to identify what skills and knowledge are needed to be successful as a 
nurse [22]. Studies have shown that people tend to overestimate their abilities as compared to actual performance, especially if they are performing poorly, and it is these individuals that do not have selfawareness of their lack of [23]. Additionally, studies focused on self-assessment have shown that people's overrated self-perception indicated overconfidence in newly learned abilities $[5 ; 23 ; 24]$. Cato, Lasater, \& Peeples [25] used the Lasater Clinical Judgment Rubric (LCJR) as a self-assessment tool with students following their simulation experiences and determined it effectively described student progress and furnished a framework for students to organize their thoughts about managing patient situations. Awareness of their developing abilities, rather than an assumption of accomplishment is key for maintaining patient safety.

\subsection{The LCJR}

The (LCJR) is a rubric developed to provide nursing students with quantified feedback on their clinical judgment abilities during simulation, an option for self-assessment, and support for those involved with evaluation of clinical thinking $[7 ; 26]$. The LCJR offers language to clearly define the four stages of clinical judgment; noticing, interpreting, responding, and reflecting, along with the overarching aspects of development from beginning, developing, accomplished and exemplary [7]. The aspect of Effective Noticing is comprised of the three dimensions or clinical indicators of focused observation, recognizing deviation from expected patterns, and information seeking. The aspect of Effective Interpreting is made up of the dimensions of prioritizing data, which focuses on the most relevant data needed to explain the patient's condition, and making sense of the data, which includes the use of heuristics to compare this situation's patterns with known patterns to develop an effective plan of care. The aspect of Effective Responding contains the dimensions of a calm and confident manner, clear communication, well planned interventions, and being skillful. Effective Reflecting consists of evaluation and self-analysis and a commitment by the student to improve [7]. The rubric has been shown to foster the development of clinical judgment and provides a mechanism for identifying gaps in the student's knowledge [7].

\subsection{Virtual Community}

A virtual community is a web-based application which features a community of fictional characters that can provide contextualization of nursing education [27]. The characters' stories in the VC represent a broad scope of health issues within the context of diverse backgrounds. The VC has been shown to contribute to the development of clinical judgment abilities through the addition of familiarity of the characters which contribute to the knowing or understanding of the character as a person and of the character's patterns that are unique to them. Additionally it has been found to increase student engagement and add complexity and confounding factors to the characters' situation [28].

\section{METHODS}

Two faculty members were designated to develop and implement the remediation programs. These faculty had extensive experience with clinical nursing education and simulation development. Instructors who taught clinical practicums were informed to refer students to the remediation program who were struggling with clinical judgment abilities and not meeting the clinical performance objectives. The referral identified the student's course level, strengths, and areas needed for remediation in clinical judgment. Students were not required to participate in remediation, but were aware they were not meeting clinical performance objectives. All students referred opted to participate and complete the remediation program.

The remediation faculty provided each student with the VC character information needed for them to prepare for their initial evaluation simulation, just as they would do prior to an inpatient hospital clinical experience. Our School of Nursing has a repository of low and high fidelity simulations that have been created by content experts and validated by simulation experts. A number of these simulations are based on characters from The Neighborhood $2.0^{\mathrm{TM}}$, a commercially available VC published by Pearson Education. These were the ones chosen for the remediation program. Students were already familiar with the VC characters as they were used in several nursing courses, as well as in the nursing fundamental skills lab. This familiarity facilitated student comfort with the simulation and promoted contextual application. In order to determine the student's baseline clinical judgment abilities, the simulation chosen for the initial evaluation focused on the deficits identified in the 
clinical instructor referral. The student performed in the simulation with the remediation faculty present and available to assist the student if they became confused or had questions.

At the completion of the initial evaluation simulation but prior to debriefing, the student was introduced and oriented to the LCJR and asked to rate their own simulation performance on each aspect of the rubric, basing their rating choices on their assessment of their own judgment abilities. Remediation faculty separately completed the LCJR and scored the students actual performance on the rubric. The LCJRs were scored within the aspects of clinical judgment: noticing, interpreting, responding, and reflecting, and then categorized at a beginning, developing, accomplished, or exemplary level of performance. The two remediation faculty members had previous experience using the LCJR, and had inter-rater reliability, through previous practice with scoring students in simulation. A thorough debriefing followed which used the LCJR as the basis for highlighting student strengths and weaknesses in clinical judgment. Additionally the LCJR's ratings by the faculty and student were compared, which enabled a thorough discussion of the cues that were missed, the information seeking that was needed, the prioritizing done incorrectly, the communication abilities required, the interventions missed, and the assessments not completed.

Based on the results of the initial evaluation simulation, an individualized learning plan with suggested remediation activities was developed for each student. The plan identified strengths, weaknesses, and areas of their clinical judgment that required remediation. The LCJR provided a clear picture of the areas of clinical judgment in which to focus the remediation, with the overarching goal to help the student to successfully achieve the clinical objectives. The majority of plans included some combination of the concepts of communication, organization, clinical judgment, and psychomotor abilities. Resources needed to complete the remediation activities were made available to students, but they were informed it was their responsibility to follow the plan as suggested. Both the student and referring clinical instructor received a written evaluation of the student's initial simulation performance, along with the individualized learning plan.

The student was given approximately two weeks after the initial evaluation simulation to take advantage of the remediation activities identified on the individualized learning plan. Then the remediation faculty provided each student with the VC character information needed for them to prepare for their final evaluation simulation. This low or high fidelity simulation was designed to showcase the student's progress in their clinical judgment abilities, so they completed it without assistance of any type. At the conclusion of the simulation, the student and remediation faculty separately completed the LCJR to determine the level of clinical judgment abilities. Debriefing occurred at the end of the experience using the same format as the initial simulation. A comparison of the faculty and student LCJR scores was also discussed. A final written report was completed and given to both the student and clinical instructor. Recommendations were made by the remediation faculty to the student for continued improvement in clinical judgment abilities. The final evaluation simulation did not indicate course progression or failure, but was meant to describe summative progress in the student's clinical judgment abilities.

\section{RESUlTS}

The multi-modal approach to remediation was implemented in our undergraduate baccalaureate program. The evaluation of this program was based on anecdotal feedback from faculty and students to identify the strengths, limitations, and plans for future amelioration.

Referring clinical instructors $(n=5)$ and participating students $(n=5)$ were very favorable of the program overall, with all of the clinical instructors indicating their students had shown improvement in their clinical judgment abilities. Sixty percent of the students gained enough improvement to achieve the clinical performance objectives. Students unanimously believed that they had improved in their clinical judgment abilities. Additionally, students indicated that the program assisted them in recognizing their strengths and weaknesses, and commented that through the use of the VC they recognized the value of knowing the patient's patterns or responses that were unique to that person. The remediation program confirmed for two students that progression in the nursing program was not in their best interest.

The LCJR scores after the initial evaluation simulation indicated that in general the students' selfassessed their performance at the Accomplished level while the faculty rated the student's actual performance at the Beginning level, moving toward the Developing level. Students rated themselves 
highest in the areas of noticing and responding while faculty scored them lowest in the same areas. Although having confidence in one's ability may be seen as a positive, it may also be indicative of rash judgments and flawed thinking as the overconfident student comes to a conclusion very quickly, perhaps erroneously, without gathering enough information.

After the final evaluation simulation, the LCJR scores showed that in general the student's selfassessed ratings had decreased to the Developing level and the faculty's rating of the student's actual performance remained between the Beginning and Developing levels. Student's ratings in all areas of clinical judgment declined while faculty's scores stayed similar to the initial scoring. While an improvement in clinical judgment abilities was not readily visible with the rubric, a decrease in the gap between self-assessment and actual performance was apparent. As students came to understand the actual level of their clinical judgment abilities, they became more self-aware of their strengths and deficits, which resulted in clinical judgment abilities which were more in tune with their actual performance. Studies have shown that self-assessment is an essential skill to master and cited that when self-assessment is combined with a realistic performance assessment, the variation between the two diminishes and overall performance is maximized [5; 29]. Periodic self-assessments and performance assessments over time were shown to improve self-accuracy which was further enhanced with the use of peer assessment and benchmarking [5;23].

\section{DISCUSSION}

The use of a multi-modal approach appeared to successfully meet the student's learning needs while using a pedagogically sound framework. The individualized learning plan, simulation that utilized a VC, self-assessment of performance and use of the LCJR, provided a clearly defined process that incorporated factors that have been shown to enhance clinical judgment abilities. Early identification of struggling students combined with a multi-modal approach to remediation increased the opportunity for the learning that led to student success in meeting clinical performance objectives. Simulation allowed students to gain experience while in a realistic learning environment that allowed for the practice of clinical judgment abilities without the fear of patient harm. Use of the VC allowed students to see the simulation patients as individuals with their own unique responses to the situation. Students had the opportunity to see how the character as a person and recognize the character's patterns, unique to them. By adding this patient centric element to the abilities experience, remediation faculty and clinical instructors felt that students were better able to remember and apply what they had learned and practiced in simulation to similar patients they encountered in the clinical setting. Having experience with situations similar to the one displayed in the simulated activity could contribute to an increase in the knowing of the patient and of the pattern of patient responses for this type of event, which would aid experienced nursing in using the heuristic of pattern recognition to better respond to the simulation $[9 ; 30]$.

As a means to help decrease the gap between the self-assessed judgment scores and the actual performance ratings, students should perform self-assessment after every simulation or significant event with patient care. Studies indicate that with use of the LCRJ as a self-assessment tool, the students' self-assessment often parallels the instructor's assessment [25]. This should be followed each time by a detailed debriefing with the instructor who evaluated their actual performance. During this time of reflection, the simulation should be broken down in detail examining what clinical judgment abilities were used and comparing them to what was needed or missed. Studies have shown that self-assessment is an essential skill to master, improves with repeated opportunities to practice, and when combined with a realistic performance assessment, the variation between the two diminishes and overall performance is maximized [5]. The LCJR offers evaluators a tool that facilitates high level debriefing which aids students in a thorough reflection of their nursing care. It also provides a vehicle for self-assessment of clinical judgment abilities [31;32]. This program utilized the LCJR to provide self-assessed and faculty feedback on clinical performance at the end of each simulation. The discrepancy between faculty and student LCJR scores diminished at the end of the remediation program demonstrating a more accurate self-assessment of actual performance. Awareness of their developing clinical judgment abilities, rather than an assumption of accomplishment is vital for maintaining patient safety.

Based on the feedback and apparent usefulness of this multi-modal remediation approach, an elective course has been created for students who are struggling to function competently in the clinical setting. This course will use the foundational elements of the multi-modal remediation approach, and will 
have the luxury of having a longer time frame in which to help students enhance their clinical judgment abilities.

\subsection{Limitations}

This program was used with a small number of students in a large, university setting and therefore results are not generalizable. Student unfamiliarity with the LCJR may have yielded inaccurate selfassessments of their clinical performance. As the program continues the simulations should be videotaped which would allow students to reflect visually on their actual performance. The aspects of the LCJR will be compared to the video for an accurate look at clinical judgment abilities.

\section{CONCLUSION}

Using a multi-modal approach to remediation was an effective way to structure this program as a means to incorporate strategies to improve clinical judgment abilities. The individualized learning plan provided the student with a clear action plan to enhance their clinical judgment abilities. Through the use of simulation students were able to gain experience and enhance their judgment abilities in a safe environment. Student self-assessment of their simulation performance compared to actual performance as evaluated by the remediation faculty provided a way for students to have a more accurate awareness of the level of their clinical judgment abilities. The LCJR worked well as a tool for student self-assessment and for evaluation of clinical judgment abilities with simulation. The rubric provided a means for facilitating reflection on performance and identified gaps in the student's clinical judgment. The VC contributed to the enhancement of clinical judgment abilities by aiding the students in the knowing or understanding of the character as a person and their unique patterns related to their illnesses. This clinical remediation program provided an opportunity to enhance clinical judgment abilities which allowed students who would have otherwise been unsuccessful with their nursing education, a chance for success.

\section{REFERENCES}

[1] Gallant, M., MacDonald, J., and Higuchi, K.A., High-fidelity patient simulation: Considerations for effective learning, Nurs. Educ. Perspect. 31(5), 223-227 (2006).

[2] Lasater, K. and Nielsen, A., The influence of concept-based learning activities on students' clinical judgment development, J. Nurs. Educ. 48(8), 441-446 (2008).

[3] P. Benner, P.E. Benner, C.A. Tanner, and C.A., Chesla, Expertise in Nursing Practice: Caring, Clinical Judgment \& Ethics, $2^{\text {nd }}$ ed. New York, NY: Springer, (2009).

[4] Gillespie, M. and Pattersen, B.L., Helping novice nurses make effective clinical decisions: The situated clinical decision-making framework, Nurs. Educ. Perspect. 30(3), 164-170 (2009).

[5] Sitzmann, T. and Johnson, S.K., When is ignorance bliss? The effects of inaccurate selfassessments of knowledge on learning and attrition, Organ. Behav. Hum. Decis. Process. 117, 192-207 (2012).

[6] Tanner, C. A., Thinking like a nurse: A research-based model of clinical judgment in nursing, J. Nurs. Educ. 45(6), 204-211 (2006).

[7] Lasater, K., High-fidelity simulation and the development of clinical judgment: Students' experiences, J. Nurs. Educ. 46(6), 269-276 (2007).

[8] P. Benner, M. Sutphen, V. Leonard, and L. Day., Educating Nurses: A Call for Radical Transformation. San Francisco, CA: Jossey-Bass, (2010).

[9] Tanner, C.A., Benner, P. Chesla, C. and Gordon, D.R., The phenomenology of knowing the patient, J. Nurs. Scholarsh. 25(4), 273-280 (1993).

[10] Haskvitz, L and Koop, E., Students struggling in clinical? A new role for the patient simulator, J. Nurs. Educ. 43, 181-184 (2004).

[11] Lynn, M.C. and Twigg, R.D. A new approach to clinical remediation, J. Nurs. Educ. 50(3), 173175 (2011).

[12] Nielsen, A., Noone, J., Voss, H., and Mathews, L. Preparing nursing students for the future: An innovative approach to clinical education, Nurse. Educ. Pract. 13(4), 301-309 (2013).

[13] Evans, C.J. and Harder, N., A formative approach to student remediation, Nurse Educ. 38(4), 147-151 (2013). 
[14] C. Hamilton and A.H. Morris, Transformative Learning in Simulated Environments. In Morris, A.H. \& Faulk, D.R., eds. Transformative Learning in Nursing: A Guide for Nurse Educators. NY, NY: Springer Publishing Company, 2012, pp. 107-118.

[15] Cullieton, A. L., Remediation: A closer look in an educational context. Teach, Learn, Nurse, 4, 22-27 (2009).

[16] Bambini, D., Washburn, J, and Perkins, R., Outcomes of clinical simulation for novice nursing students: Communication, confidence, clinical judgment. , Nurs. Educ. Perspect. 30(2), 79-82 (2009).

[17] Bensfield, L, Olech, M., and Horsley, L., Simulation for high-stakes evaluation in nursing, Nurse Educ. 37(2), 71-74 (2012).

[18] Garrett, B., MacPhee, M., and Jackson, C., High-fidelity patient simulation: Considerations for effective learning, Nurs. Educ. Perspect. 34(5), 309-313 (2010).

[19] Wolf, L., Dion, K., Lamoureaux, E., Kenny, C., Curnin, M., Hogan, A., Roche, J., and Cunningham, H., Using simulated clinical scenarios to evaluate student performance, Nurse Educ. 36(3), 128-134 (2011).

[20] Banning, M., The think aloud approach as an educational tool to develop and assess clinical reasoning in undergraduate students, Nurse Educ. Today. 28(1), 8-14 (2008).

[21] Benner, P., Stannard, D., and Hooper, P.L., A "thinking-in-action" approach to teaching clinical judgment: A classroom innovation for acute care advanced practice nurses, Adv. Pract. Nurs. Q. 1(4), 70-77 (1996).

[22] Duchscher, J. B., A process of becoming: The stages of new nursing graduate professional role transition, J. Contin. Educ. Nurs. 39(10), 441-450 (2008).

[23] Dunning, D., Heath, C., and Suls, J.M., Flawed self-assessment: Implications for health, education, and the workplace, Psychol. Sci. Public Interest. 5(3), 69-106 (2004).

[24] Fenske, C., Harris, M., Aebersold, M., and Hartman, L., Perception versus reality: A comparative study of clinical judgment abilities in nurses during a simulated activity, J. Contin. Educ. Nurs. 44(9), 399-405 (2013).

[25] Cato, M.L., Lasater, K., and Peeples, A.I., Nursing students' self-assessment of their simulation experiences, Nurs. Educ. Perspect. 30(2), 105-108 (2009).

[26] Adamson, K., Gubrud, P., Sideras, S., and Lasater, K., Assessing the reliability, validity, and use of the Lasater clinical judgment rubric: three approaches, J. Nurs. Educ. 51(2), 66 (2012).

[27] Giddens, J., Fogg, L., and Carlson-Sabelli, Learning and engagement with a virtual community by undergraduate nursing students, Nurs. Outlook. 58, 261-267 (2010).

[28] Gonzalez, L. and Fenske, C., The use of virtual community to contextualize learning activities for nursing education, J. Nurs. Educ. 51(1), 38-41 (2012).

[29] Forsterling, F., \& Morgenstern, M., Accuracy of self-assessment and task performance: Does it pay to know the truth? J. Educ. Psychol. 94(3), 576-585 (2002).

[30] Lanuza, D., Hicks, F., Simmons, B., Holm, K., and Fonteyn, M, Clinical reasoning in experienced nurses, West J. Nurs. Res. 25(6), 701-719 (2003).

[31] Lasater, K., Clinical judgment: The last frontier for evaluation, Nurs. Educ. Pract. 11, 86-92 (2011).

[32] Nielsen, A., Stragnell, S., and Jester, P., Guide for reflection using the clinical judgment model, J. Nurs. Educ. Pract. 13(4), 301-309 (2013). 


\section{AUTHORS' BIOGRAPHY}

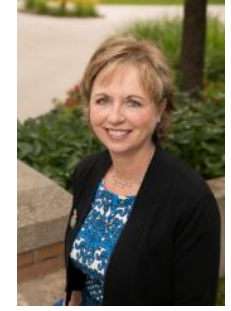

Dr. Cynthia Fenske, is the Campus Dean for Nursing and Associate Professor at Concordia University Ann Arbor in Ann Arbor, Michigan. She is also a Clinical Assistant Professor Emeritus from the University of Michigan School of Nursing in Ann Arbor, Michigan. Dr. Fenske's research interests aim to explore innovative teaching strategies and methods that teach and enhance clinical judgment skills in undergraduate nursing students. She is heavily involved with teaching and mentoring faculty in the creation and implementation of active learning techniques in the classroom and in the development and integration of simulation throughout the nursing curriculum.

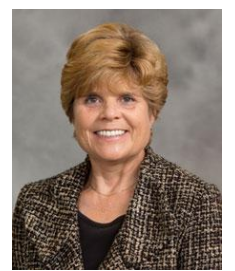

Dr. Deborah Price, is a Clinical Assistant Professor at the University Of Michigan School Of Nursing in Ann Arbor, Michigan. Dr. Price seeks to contribute to the transformation of nursing education so as to prepare confident and competent nursing graduates who are ready to transition to professional practice. She utilizes innovative technology methods and strategies in nursing education to promote student learning and engagement. Dr. Price seeks to impact the quality of care that patients receive in chronic illness and end-of-life situations. Additionally, she advocates for informed patient and family decision-making in advanced illness situations. Her research focus is on interdisciplinary palliative care competencies. 